\title{
Coaching como herramienta gerencial: reflexiones teóricas
}

\author{
Podesta Gavilano, Luis Enrique ${ }^{1}$ \\ Vigo Ayasta, Elsa Regina ${ }^{2}$ \\ Ponce Yactayo, Dora Lourdes ${ }^{3}$ \\ Romero Vela, Sonia Lidia ${ }^{4}$
}

\section{Resumen}

A nivel mundial las organizaciones indistintamente del sector en el cual se encuentran inmersas, requieren de la implementación de estrategias gerenciales que le permitan garantizar el cumplimiento de objetivos y metas fundamentales en su gestión organizacional. Así, en el campo gerencial surgen estrategias que coadyuvan a los niveles directivos a lograr desempeños exitosos en su personal y en su organización, valiéndose para ello en el desarrollo de competencias, conductas, habilidades y actitudes que permitan la potenciación de recursos, a la vez que propicien una mejor calidad de vida y mayor satisfacción con la práctica de la actividad profesional cotidiana.Surge entonces el coaching como estrategia gerencial, que se traza el fomento del crecimiento personal y profesional del ser humano y busca instaurarse como una filosofía de vida en las personas. En este sentido, el objetivo de este artículo es describir el coaching como herramienta gerencial en las organizaciones, para ello se empleó una metodología cualitativa basada en la revisión bibliográfica y de documentos. Se precisan como resultadosque el coaching posee características y dimensiones que le permiten a la gerencia de las organizaciones avanzar y mejorar sus estrategias para alcanzar los objetivos planteados. Finalmente, se concluye que el coaching es una herramienta útil que toda organización debería implementar en su quehacer cotidiano.

Palabras clave:coaching; herramienta gerencial; organizaciones

Recibido: 20.03.19 Aceptado: 20.06.19

1 Doctor en ciencias de la salud. Docente principal de departamento de medicina de la facultad de medicina de San Fernando y de la escuela de postgrado de la Universidad Nacional Mayor de San Marco. E-mail: Ipodestag@unmsm.edu.pe. Orcid: 0000-0003-0122-8835

2 Docotora en Salud publica. docente de la escuela universitaria de postgrado de la Universidad Nacional Federico Villareal. reginavigo27@gmail.com. Orcid: 0000-0002-4090-8887

3 Doctora en administración de la educación. Docente de la escuela de Postgrado de la Universidad Privada Cesar Vallejo. E-mail: doraponce33@hotmail.com. Orcid: 0000-0001-7823-6839

$4 \quad$ Magister en Psicologia educativa. docente de la escuela de postgrado Universidad Privada Cesar Vallejo. E-mail: sirv131979@hotmail.com. Orcid: 0000-0002-9503-410X 


\section{Coaching as a management tool: theoretical reflections}

\section{Resumen}

A nivel mundial las organizaciones indistintamente del sector en el cual se encuentran inmersas, requieren de la implementación de estrategias gerenciales que le permitan garantizar el cumplimiento de objetivos y metas fundamentales en su gestión organizacional. Así, en el campo gerencial surgen estrategias que coadyuvan a los niveles directivos a lograr desempeños exitosos en su personal y en su organización, valiéndose para ello en el desarrollo de competencias, conductas, habilidades y actitudes que permitan la potenciación de recursos, a la vez que propicien una mejor calidad de vida y mayor satisfacción con la práctica de la actividad profesional cotidiana.Surge entonces el coaching como estrategia gerencial, que se traza el fomento del crecimiento personal y profesional del ser humano y busca instaurarse como una filosofía de vida en las personas. En este sentido, el objetivo de este artículo es describir el coaching como herramienta gerencial en las organizaciones, para ello se empleó una metodología cualitativa basada en la revisión bibliográfica y de documentos. Se precisan como resultadosque el coaching posee características y dimensiones que le permiten a la gerencia de las organizaciones avanzar y mejorar sus estrategias para alcanzar los objetivos planteados. Finalmente, se concluye que el coaching es una herramienta útil que toda organización debería implementar en su quehacer cotidiano.

Palabras clave:coaching; herramienta gerencial; organizaciones

\section{Introducción}

A nivel mundial las organizaciones indistintamente del sector en el cual se encuentran inmersas, requieren de la implementación de estrategias gerenciales que le permitan garantizar el cumplimiento de objetivos y metas fundamentales en su gestión organizacional. En el contexto actual, marcado por regulaciones y exigencias, dichas instituciones, deben centrarse en la definición de objetivos pertinentes y ajustados a las realidades actuales. Ello exige el desarrollo y ejecución de planes con alto sentido de responsabilidad social, cuyo beneficio plausible sea evidente en la sociedad en la cual se encuentran inmersas.

Así, en el campo gerencial surgen estrategias que coadyuvan a los niveles directivos a lograr desempeños exitosos en su personal y en su organización, valiéndose para ello en el desarrollo de competencias, conductas, habilidades y actitudes que permitan la potenciación de recursos, a la vez que propicien una mejor calidad de vida y mayor 
satisfacción con la práctica de la actividad profesional cotidiana.

Dentro de estas estrategias gerenciales, se debe mencionar el coaching que se traza el fomento del crecimiento personal y profesional del ser humano y busca instaurarse como una filosofía de vida en las personas. Esta herramienta se convierte en esencia fundamental para superar situaciones adversas en una sociedad que demanda grandes compromisos y exigencias. Convirtiéndose cada actuación, en un sentir responsable con la realidad circundante con la que interactúan las organizaciones, en este caso las dedicadas al campo educativo, cuya responsabilidad se acentúa al ser responsables de la educación de niños y adolescente con valores y principios arraigados y altos niveles de calidad.

En la actualidad, la gerencia empresarial ha sido quizás la más interesada en aplicar modelos organizacionales, a fin de lograr mayor eficacia y efectividad respecto a la productividad, con el fin de respaldar entrenamientos, que generen en los individuos cambios óptimos, tanto de índole personal como institucional. De allí, que el punto clave de todo este proceso de retroalimentación, está en que las personas pertenecientes a una organización, conozcan a profundidad sus valores y principios y éstos sean coherentes con los de sí mismos, de tal manera que exista una especie de simbiosis entre ambas partes. Por tales motivos expresados se hace necesario mencionar lo que se afirma Molano (2005:4):

El cambio organizacional...
es un cambio de actitudes, un
cambio humano individual y
colectivo, íntimamente ligado a
las características personales del

gerente y a la de los otros individuos que lo designen a él, o que a su vez son escogidos por él como sus colaboradores.

Para lograr lo anterior, el enfoque organizacional llamado Coaching, se basa en un sistema de liderazgo integral, que plantea un conjunto de principios, de direccionamiento y movilización humana y empresarial hacia el logro de resultados, conjugados de una manera especial y diferenciada, con herramientas y técnicas específicas para la identificación de talentos, técnicas de entrenamiento y desarrollo de competencias de productividad en el trabajo, estableciendo así mismo, valores, normas de disciplina, estrategias y tácticas orientadas al mejoramiento del desempeño de los integrantes de la organización, liderando como equipo mediante el modelaje.

Lo anteriormente expuesto conduce al planteamiento del objetivo de este trabajo, el cual consiste en describir el coaching como estrategia gerencial en las organizaciones, Para ello, se asume una metodología cualitativa descriptiva basada en revisiones bibliográficas y documentos que muestran el estado del arte.

\section{Coaching: consideraciones teóricas y cualidades esenciales}

Antes de indagar sobre el coaching como estrategia gerencial, es necesario definirlo, de allí que desde una perspectiva etimológica, el término "coach" procede del idioma inglés; y proviene del vocablo de origen húngaro, kocsi; puesto de acuerdo a Bou (2012) y Larez (2008), dicho término se popularizó en la ciudad húngara de Kocs para los siglos $\mathrm{XV}$ y $\mathrm{XVI}$; y el término 
era empleado para denominar un tipo de carruaje originario de un pueblo del mismo nombre.

Tal carruaje, para Bou (2012) era caracterizado por ser el único provisto de un sistema de suspensión. En esta ciudad se comenzó a hablar del carruaje de Kocs, símbolo de la excelencia. De esta forma Kocsi(pronunciado cochi) se trasladó al alemán como Kutshe, al italiano como cocchio $y$ al español como coche. De esta forma, se puede decir que la palabra coach, es de origen húngaro y hace referencia a un vehículo tirado por animales que servía para transportar personas de un lugar a otro.

En el campo de la gerencia, el término coach de acuerdo con Colorado y Corcino (2015), surge en Estados Unidos y Canadá, a finales del siglo $X X$, de la mano de John Whitmore, considerado su precursor en el campo empresarial, donde ha tenido una considerable incidenciay aceptación.

También, refieren Sánchezy Boronat(2014), que dicho término es utilizado por primera vez en el año de 1830 en la Universidad de Oxford, para denotar un instructor o un entrenador que "transporta" a un estudiante a través de un examen. A partir de entonces, el término coaching ha sido utilizado para describir el proceso de transportar personas desde donde están, hasta donde desean estar.

Así, este concepto, que ha venido impregnando el quehacer diario; según Richardson (2005:73), es visto como un término que al igual que la palabra "training" se traduce como entrenamiento, pero con la connotación de lo que hace un entrenador de campeones. Definiendo el autor, un "método fundamentado en dirigir, instruir y entrenar a una persona o a un grupo de ellas, con el objetivo de conseguir alguna meta o desarrollar habilidades específicas". En este sentido, se puede precisar su conceptualización como el arte de trabajar con los demás para que ellos obtengan resultados fuera de lo común y mejoren su desempeño, es decir, es un proceso que ayuda a las personas a desarrollar su verdadero potencial y a introducir cambios en su estilo de vida.

De lo referido, el coaching como estrategia gerencial según Salazary Molano (2009) es un sistema que incluye conceptos, estructuras, procesos, herramientas de trabajo e instrumentos de medición y grupos de personas. Comprende un estilo de liderazgo en forma particular de seleccionar la gente o crear grupos de personas en desarrollo. De acuerdo con los autores, el coaching se ha convertido en un conjunto de principios de direccionamiento $y$ movilización humana y empresarial hacia el logro de resultados.

Consideran, que a nivel gerencial se conjuga de una manera especial y diferenciada con herramientas y técnicas específicas para la identificación de talentos; ofrece técnicas para el entrenamiento y desarrollo de competencias para la productividad en el trabajo, el acompañamiento de la gente, y la creación de espíritu de trabajo en equipo. Asimismo, Amador (2009), lo concibe como unproceso de aprendizaje continuo, donde el coach trabaja con su asesorado en una relación de mutuo interés, para alcanzar una mejor versión de sí mismo, todo esto con el fin de lograr metas y resultados, más allá de lo que el asesorado espera en un principio. Lo que se busca con el coaching es potenciar y desarrollar las capacidades de una persona o grupos de personas para lograr objetivos concretos.

En lo que respecta a las 
cualidades del coach, según señala Dessler (2009:107) "son características y competencias personales que llevan a un individuo a tener una experiencia exitosa en el desempeño de la función gerencial". De allí, que todo buen gerente debe poseer ciertas cualidades que servirán para medir el cumplimiento de su rol en el puesto cuya acción dependerá de sus cualidades físicas, personales, sociales o sensoriales y que implican algún potencial que la persona desarrolla en su trabajo.

De lo anterior, se puede indicar tal como lo sostiene Bou (2012)que por constituirse el coaching en una filosofía de vida, se convierte en un método creativo que pretende ayudar a revisar la visión que se tiene del mundo en pro de mejoras individuales y sociales; en este sentido todo coach debe poseer cualidades para acompañar a los individuo o grupo de personas; induciéndolos a profundizar el conocimiento de sí mismo y guiarlo en el desarrollo de nuevas acciones; con el objeto de mejorar su desempeño personal y profesional y así poder obtener resultados óptimos en sus metas de vida.

De igual manera, ColoradoyCorcino (2015),señalan que esta herramienta que persigue ayudar a identificar los atributos y destrezas que posee una persona, a la vez de facultarla para que pueda desarrollarlas; demanda del gerente educativo que sea una persona capaz de generar procesos interactivos y sistemáticos de aprendizaje; para llegar al descubrimiento del potencial individual, centrado en el presente y orientado al cambio y la excelencia.

Entre las principales cualidades, se encuentran:

\section{- Saber escuchar}

Para Máñez, Navarro y Bou (S/F), el saber escuchar consiste en comprender el punto de vista de la otra persona, cómo piensa y siente, y cómo ve y va por el mundo. Por su parte, Goleman (2000), define el saber escuchar como escuchar bien, profundamente; significa ir más allá de lo que se dice, hacer preguntas y repetir con palabras propias lo que se ha oído, a fin de asegurarse de haber entendido bien. Al encaminar dichas acciones, se escucha activamente.

En este sentido, el saber escuchar, implica "escuchar" íntegra y atentamente el mensaje del emisor y "devolverle" lo que pensamos haber recibido. Escuchar parece algo sencillo; sin embargo, prestar verdaderamente atención y escuchar con atención, constituye una experiencia en verdad muy profunda (Máñez, Navarro y Bou (S/F).

Saber escuchar y atender es un requisito primordial del coach, que puede afectar, si no se tiene, la motivación y la falta de atención, así como la posibilidad de comprender a éste erróneamente y elegir una estrategia de análisis inadecuada. Para Máñez, Navarro y Bou $(\mathrm{S} / \mathrm{F})$, existen cuatro niveles de escucha:

1)Oír:se ubica en el nivel más superficial de escucha. Puedes estar oyendo a alguien y al mismo tiempo estar pensando o haciendo otra cosa. Para oírle no necesitas prestarle atención. El coach no debería estar nunca en este nivel;

2) Escuchar a: es el segundo nivel que consiste en escuchar, pero con una pregunta en mente, ¿qué significa esto para mí?. Escuchas desde dentro de tu propia experiencia, utilizando la experiencia de esta otra persona para activar tus propios recuerdos. Se trata del nivel cotidiano de escucha, adecuado 
para las conversaciones ordinarias pero no para el coaching;

3) Escuchar para: se corresponde a escuchar buscando algo en lo que el otro dice. El coach puede tener una idea en mente, filtra lo que el plantea el hablante y lo selecciona para formarse una opinión. Para ello es probable que el coach establezca un diálogo interno consigo mismo; y

4)Escucha consciente o activa: consiste en una escucha profunda con un mínimo de juicio. Te mantienes al margen. Hay un mínimo de dialogo interior. Es el nivel en que tu intuición puede funcionar mejor. Esta escucha tiene tres enemigos: a) diálogo interno: si te escuchas a ti mismo no puedes escuchar al emisor. Así pues, deja que sea el emisor quien te hable y no tú mismo; b) tensión muscular: no es fácil escuchar si estás tenso, de modo que si descubres que tu atención disminuye, relájate. Asegúrate de estar cómodo, la incomodidad física es otro factor de distracción; y c) Mirada fija: tu mente estará más abierta y receptiva si utilizas todo el campo visual, de modo que suaviza tu enfoque y amplia tu visión. Permanece todo el tiempo que puedas con la visión amplia y desenfocada.

En este sentido, es la escucha activa o consiente es una cualidad del verdadero coach cuyo propósito es liderar procesos de cambio tanto en equipos de trabajo como en las personas que participan en ellos. Esta cualidad del coaching, asume una serie de principios, los cuales según Pascal, D. (2000:4748), se orientan a: la aceptación de opiniones, la tolerancia y el respeto a opiniones contrarias, la expresividad, empatía, el silencio, la disposición, evitar acusaciones y manipulaciones, agregando Máñez, C., Navarro, B. y Bou, F., (S/F): la concentración en el tema, la motivación por la escucha, una actitud mental positiva, objetiva y constructiva, la creación de un clima de confianza.

Ambos autores, persiguen dejar claro los aspectos valorados al poner en práctica una verdadera escucha activa. Estos principios, sin duda guiarán un verdadero proceso de comunicación entre el coah y sus colaboradores, favoreciendo un intercambio positivo entre ellos. Para finalizar plantea Goleman, D. (2000: 68), que para triunfar en el lugar de trabajo es esencial escuchar bien.

\section{- Credibilidad}

En lo que respecta a la credibilidad, esta cualidad, asociada de manera directa con la confianza juega un rol fundamental al momento de ejercer el coahing. De acuerdo con Kouzes, J. y Posner, B. (2006), resulta ser una palabra de amplio contenido, pues va desde "descubrirse a usted mismo", hasta "alentar la esperanza". Para los autores, la credibilidad trata el modo en los líderes se gana la confianza de sus poderantes. Es una cualidad que la gente admira en los líderes, marcando una gran diferencia entre conductas y desempeño laboral. Esta cualidad del coach, tiene efectos significativamente positivos sobre el desempeño individual y organizacional; se basa en las cualidades de ser honesto, competente e inspirador, siendo sumamente importante en esta era de cambios organizacionales y competencia internacional.

Para los autores, de todos los atributos de la credibilidad, la dimensión de la honestidad es responsable de más variaciones en la confiabilidad que de cualquiera de los otros factores combinados. Ser considerado confiable, integro, honesto y veraz es algo 
esencial. La credibilidad se gana con el tiempo. No llega automáticamente con el empleo o títulos. Se inicia temprano en nuestras vidas y carreras. Se constituye en la base del liderazgo y de todas las relaciones que funcionan correctamente; está determinada por los poderantes y se constituye en fundamento y cimiento del liderazgo; siendo una base sólida para líderes.

Para Bayo (2014), si deseas ser un Coach exitoso, se debe construir confianza y una imagen de credibilidad; ambos serán el mejor patrimonio y activo: confianza, para que las empresas deleguen en ti la responsabilidad de implementar las técnicas del coaching; y credibilidad para que la gente se sienta tranquila y motivada con tus propuestas y actividades. La credibilidad se gana asumiendo metas realistas y no ideales inalcanzables.El Coach sabe que su imagen de credibilidad y confianza le permitirá llegar lejos, lograr que los clientes le recomienden a sus amigos y conocidos, desarrollen vínculos y relaciones a largo plazo, y estén dispuestos a invertir en tus ideas, en tiempo y dinero, en recursos y gente (Bayo, 2014). Es decir, los coach que gozan de credibilidad, poseen la cualidad necesaria para ganarse el respecto de sus colaboradores, quienes confían en el cómo en sus planteamientos.

\section{- Liderazgo}

El liderazgo se considera proceso fundamental en las organizaciones, y más cuando se conducen esfuerzos de grandes grupos sociales, con culturas, pensamientos $y$ acciones diversas. Diversos han sido los autores que lo han definido dicho concepto, concibiéndolo como un proceso de influencia entre líderes y seguidores para lograr objetivos organizacionales por medio del cambio. De estos autores se cita a Lussier y Achúa (2005:103);quienes definen el liderazgo como "la capacidad y el proceso mediante el cual un individuo influye sobre la conducta de los demás, con el fin de lograr los objetivos propuestos".

También, en la misma línea, Robbins y Coulter (2010) y Daft y Marcic (2006), establecen que es un arte o proceso con capacidad para influir o moldear al grupo y motivar su comportamiento hacia el logro de las metas organizacionales, precisando Griffin (2011) que esta influencia debe ser no coercitiva, pues esto no se denotaría subordinación voluntaria.

Siendo para Lorenzo (2014),una función que dinamiza un grupo o una organización para generar su propio crecimiento en función de una misión o proyecto compartido. Plantea el autor que el liderazgo no es algo consustancial a ciertos sujetos o tipos humanos. Es, más bien, una función que el grupo atribuye, según el momento y la actividad, a determinados miembros (uno o varios) del propio grupo. No hay un líder para todas las ocasiones, sino que en cada contexto y en cada situación surge la persona o personas capaces de dinamizar y buscar soluciones al grupo. En este sentido, para efecto del estudio el liderazgo se conceptualiza como una función, una cualidad y una propiedad que reside en el grupo y que dinamiza la organización. es decir, es una cualidad de coach que requiere compromiso, energía, tenacidad, iniciativa, poder, honestidad, integridad, autoconfianza, habilidades cognitivas, conocimiento de la situación, carisma, creatividad, flexibilidad, visión, patrones de comportamiento e interacción, adaptables a la situación 
y a las necesidades de los seguidores, dado que el líder inspira y arrastra a los seguidores.

En este orden de ideas, el liderazgo del coaching como modelo que ayuda a desarrollar las competencias necesarias para el profesional, se vale de las cualidades del líder para facilitar procesos de transformaciones individuales y grupales que permitan el logro de los objetivos, diseñar y coordinar talleres, cursos y seminarios para posibilitar el desarrollo personal y profesional y realizar consultoría en organizaciones. Sobre el particular, el estilo de liderazgo de un coach según Salazar y Molano (2013:39), debe presentar los siguientes elementos que se especifican en el diagrama 1.

\section{Diagrama1 \\ Elementos del estilo de liderazgo de un coach}

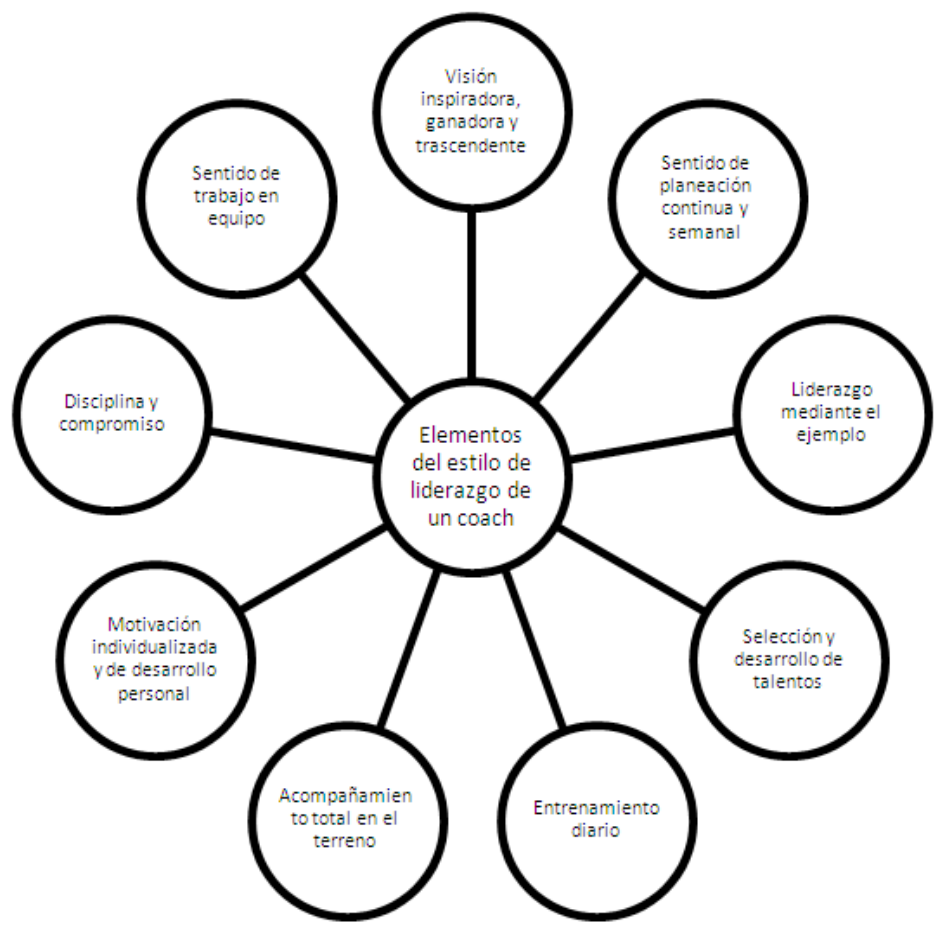

Tomado: Salazar, G. y Molano, M. (2009). Coaching en Acción. pág. 39. Editorial Mc. Graw Hill. México. 
Dichos elementos que sin duda aportaran a propiciar proceso de liderazgo de excelente nivel en las organizaciones, el disponer de líderes comprometidos, como un capital fundamental que supere la visión segmentada de ver al personal, asumiendo enfoques novedosos de dirección donde el talento humano, sus habilidades, competencias y conocimientos, se constituyen en la principal arma para afrontar los retos que se presentan.

Sobre la base de lo destacado, el liderar es ir más lejos, es sustentar una dinámica más rica y creadora con la organización y con el grupo. Es implicar, crear colaboración, buscar la satisfacción de los miembros, innovar y mejorar continuamente. Es un entrenador, facilitador, directivo que propone modelos de competencias, para educar en las virtudes propias de un verdadero líder; arraigar convicciones profundas y perennes, como la humildad, la solidaridad, el servicio a los demás, la colaboración, el amor... de tal suerte que se esté educando en una cultura de servicio.

\section{- Visión inspiradora}

La visión inspiradora y trascendente, se convierte en la primera característica de un coach Salazar y Molano (2013). Permite concebir el ideal pretendido, de llegar a ser ganador, de lograr el mejoramiento del equipo, inspirarse diariamente por lo que se hace, hasta el punto de poner todas las estrategias y tácticas para triunfar y crear una cultura ganadora. Debe ser compartida por todos, y convertirse en la visión de todos. Debe ser una visión muy alta, y a pesar que exija grandes esfuerzos, es la que permitirá lograr grandes metas.
Una visión inspiradora, según los autores citados exige definir metas personales y comunes, compromiso consigo mismo y el equipo, excelencia en el estilo de vida personal, capacidad para convocar grupos alrededor de metas comunes, proactividad, actitud positiva, comunicación, escucha activa, retroalimentación equilibrada, liderar mediante el ejemplo, construir sobre el error, ser paciente, comprender procesos y resolver conflictos, transferir habilidades técnicas y humanas para educar integralmente, reforzar constantemente, recompensas, resultados, autodominio personal y disciplina, autonomía de los miembros del equipo, flexibilidad, firmeza.

Con esta visión, el coach es capaz de abarcar una idea precisa del futuro, y de estimular con ella la imaginación y el entusiasmo tanto en su interior como en sus colaboradores. De acuerdo con Salazar y Molano (2009), exige elementos clave que se deben trabajar a profundidad, pues implican cambios de paradigmas y a nivel interno de las personas que deciden asumir el coaching como filosofía de vida. Así, todo coach deberá mantener una visión que le inspire a I logro de metas y objetivos, en beneficio de la organización y sus colaboradores.

\section{Proceso de entrenamiento de coach}

Quien entrena tiene muchas ocupaciones y responsabilidades. Deben planificar, organizar y dirigir los entrenamientos de su equipo de trabajo Salazar y Molano (2013), consideran que este proceso exige puntualizar conocimientos, habilidades, actitudes y comportamientos éticos; para señalar fortalezas y debilidades, analizar el 
cumplimiento de estrategias y tácticas, y en discusión conjunta, plantear los planes de acción para la efectividad del trabajo y el crecimiento personal del equipo.

Por otra parte, los autores definen el entrenamiento como un proceso de acompañamiento, un sistema donde el coach se convierte en un consultor, debiendo adoptar una actitud de amigo y socio con su subordinado. Exige analizar fortalezas y debilidades del equipo; discutir planes, entrenamiento, crecimiento y desarrollo; mostrar con el ejemplo técnicas óptimas de trabajo y desarrollo; transferir experiencia sobre situaciones específicas y fomentar la motivación constante hacia el cambio.

Asimismo, quien entrena debe observar habilidades y técnicas de comunicación, administrar de manera adecuada el tiempo y la información, observar actitudes de motivación y realizar retroalimentación equilibrada, estando consciente que no debe realizar el acompañamiento sin preparación, no debe tomar iniciativas ni hacer protagonismos o llamar la atención en presencia de otras personas.

Asimismo, para Salazar y Molano (2009:87), debe evitar evaluar al entrenado, regañar, presionarlo o amenazarlo, ni terminar el acompañamiento sin retroalimentación y si determinar los planes de acción a seguir. Para el desarrollo del proceso de entrenamiento, debe tenerse en cuenta, el autoconocimiento, la autocrítica, el autoaprendizaje y la autodisciplina, aspectos que se definen a continuación; y los cuales son fundamentales en todo proceso gerencial por cuanto ayuda al gerente educativo a auto conocerse.

\subsection{Autoconocimiento}

Dentro del proceso de entrenamiento del coach, se encuentra el autoconocimiento, definido como resultado de un proceso reflexivo mediante el cual un individuo adquiere noción de su persona, de sus cualidades y características. Como todo proceso, tiene en diversas fases: autopercepción, autoobservación, memoria autobiográfica, autoestima, autoaceptación.

Para Salazar y Molano (2013:69), la función de coach identifica necesidades individuales, se convierte en el consejero del desarrollo persona en lo individual y en su equipo, orientando a cada uno en el conocimiento de sus fortalezas y debilidades y muchas veces, señalar lo que cada cual no está viendo. De allí, que todo coach deberá insistir en los valores positivos presentes en cada uno y será un guía para que cada persona pueda construir su propio proyecto de vida, lo que exige paciencia, confrontación y mucha retroalimentación equilibrada. De esta forma la vida se torna en un devenir de aprendizaje y desarrollo de la persona, única responsable de sus resultados.

De igual manera, para Bennett (2013), es el autoconocimiento, el camino que conduce a conocernos globalmente y en todos los aspectos de la vida. Es un conocimiento íntimo que permite que se pueda vivir mejor con nosotros mismos y con los demás, alimentando capacidades propias y logrando transformar errores y fallos en triunfos. Para el autor, el autoconocimiento conlleva a adquirir más responsabilidad y examinar lo que hemos vivido hasta hoy para obtener una imagen realista de nosotros mismos.

Es así, como mediante este proceso, se reconocen valores $y$ capacidades, intereses y posibilidades. El autoconocimiento, es un proceso constante de acción sobre uno mismo que conlleva un efecto al reconocerse a sí mismo. Para dirigirse hacia este 
conocimiento hay tres pasos ineludibles: 1) tener conciencia de uno mismo, 2) descubriry explorar el propio pensamiento, los pensamientos y los actos, 3) observar y estudiar las experiencias pasadas, para que sirvan en el presente y, por tanto, en el futuro. Mediante estos tres pasos se puede diferenciar entre lo que se cree que uno es, lo que los demás creen que se es y lo que se es en realidad.

En este sentido, refiere Vinardi (2016) que el autoconocimiento abarca aspectos psicológicos y filosóficos del propio ser humano y su relación con los demás; también se relaciona con las esferas emocional y mental del ser humano abarcando también otras categorías como la biología y la energética. El autoconocimiento no implica solo la percepción y comprensión de los procesos fenomenológicos de la personalidad y de las relaciones entre un individuo y otro, sino de la acción de las relaciones entre el ser humano y todo lo demás que lo rodea

En síntesis, es el autoconocimiento el que permite al ser humano la interiorización de sus debilidades y virtudes, y sobre esta base emprender acciones para lograr las metas propuestas, conociendo de manera detallada cada aspecto esencial de su vida. Así todo gerente educativo para poder dirigir desde los postulados del coach a su equipo de docente y demás miembros de la comunidad educativa debe poseer un amplio autoconocimiento de sí mismo para poder ayudar a dicho equipo a auto conocerse y dirigirlos hacia el logro de los logros educacionales.

\subsection{Autocrítica}

Otro elemento de los procesos de entrenamiento del coach, es la autocrítica, aspecto que de acuerdo con el diccionario de la Real Academia Española (2016), está referido al juicio crítico que se realiza sobre obras o comportamientos propios. Pudiendo afirmar que la autocrítica es un concepto que si se hace adecuadamente ayudará a crecer y mejorar como personas, pero si se acoge su aspecto más negativo (autocrítica no constructiva) puede llegar a ser devastadora, sobre todo en el ámbito de las relaciones con los demás.

En este sentido, la autocrítica puede asumirse como la capacidad dedistinguir los propios defectos y de enfrentarlos con la firme promesa de encaminar acciones para realizar las cosas de la mejor manera posible. Tiene que ver con la capacidad de auto evaluarse y ser sincero con uno mismo. Sobre esto, y siguiendo la definición de la Real Academia Española, se puede afirmar que la autocríticaes un concepto que si se hace adecuadamente ayudará a crecer y mejorar como personas, pero si acoge su aspecto más negativo como una autocrítica no constructiva puede llegar a ser devastadora, sobre todo en el ámbito de las relaciones con los demás.

De esta manera, la autocrítica no solo se basa en la revisión de la conducta, implica también la supervisión del rendimiento en las diferentes áreas en las que la persona se desempeña, buscando con ello mejorar acciones de vida. Así, se debe asumir una autocrítica sana, como práctica consistente en ser conscientes de los propios fallos o errores, asumirlos y corregirlos, o al menos, llegar a atenuarlos en la medida de lo posible. Es como si se realizara una autoevaluación tanto de los puntos positivos como negativos de nuestras actividades, pensamientos o sentimientos, a través de la cual se comienza un proceso deaprendizajecon 
el propósito de mejorar aquella característica criticada. Este concepto se encuentra muy ligado al de superación personal.

Cabe destacarse que según Goleman (2010), la autocrítica sana constituye un instrumento muy importante para llegar a conocernos mejor y sobre esa base mejorar continuamente. Es un proceso difícil para muchas personas, al limitarse u oponerse a obtener una crítica de su desempeño, debido al carácter no cuantificable de su trabajo. Así, todo líder debe fomentar una autocrítica saludable, siendo esta capaz de mejorar su personalidad y sus acciones de trabajo, si esto ocurre de manera contraria, se afectaría el autoestima del ser humano alterando su equilibrio individual como ser humano que se interrelaciona e interactúa con otras personas, sea en el ámbito familiar o laboral.

\subsection{Autoaprendizaje}

e acuerdo con Guillermo y Alonzo (2010:67) "el autoaprendizaje es una forma de adquirir conocimientos donde la relación entre el profesor y la alumna o el alumno no es directa". Es decir, es el acto de emprender y completar la tarea de aprendizaje sin tutela, como un acto propio de auto reflexión, donde el acto o estudiante investiga por tu cuenta aquellas cosas que consideras importantes.

Sobre esta definición se considera el autoaprendizaje como una de las habilidades del ser humano. Cuando este proceso se realiza con voluntad y motivación propia, se puede estar en presencia de autoaprendizaje, el cual se define como un proceso fundamental en todo ser humano, definido como la capacidad de aprender de forma autónoma e independiente, es decir, a modo autodidacta. Un individuo racional, tiene la capacidad de evaluar vivencias, reflexionar, observar su alrededor y hacerse preguntas, capacidad que demuestra que el autoaprendizaje surge de la iniciativa propia, siendo unproceso donde el individuo adquiere conocimientos, actitudes y valores por cuenta propia, puede ser dado mediante estudios o experiencia.

Esta cualidad se hace necesaria para cualquier coach, la cual para Salazar y Molano (2009), es fundamental pues el desarrolla procesos de transferencia de conocimiento, y como líder de grupos sociales requiere conocimientos sustentados en la realidad que domina, es decir conocimiento que le oriente en la resolución de situaciones propias del contexto en el cual se inserte. En este sentido, el autoaprendizaje, se define como la capacidad de aprender de forma autónoma e independiente, es decir, a modo autodidacta.

Así de acuerdo a los autores antes referidos, quien desarrolla procesos de autoaprendizaje, debe poseer ciertas características: 1) ser una persona responsable, 2) asumir un aprendizaje permanente, 3) estudiar de manera independiente; estos elementos se constituyen en parte del éxito de este proceso que destaca características particulares del líder coach. El reimpulso constante y consistente en estos procesos sin duda potenciará el éxito sostenido en el líder dentro del ámbito organizacional. Por lo tanto, el autoaprendizaje se puede implementar en diversos ámbitos de la vida, siendo de gran importancia para la superación del ser humano, dado que lo ideal para cualquier persona es que año a año aprenda algo nuevo, sino la mente se queda estancada. 


\subsection{Autodisciplina}

Otro de los aspectos como proceso fundamental en el entrenamiento del coach, se encuentra la autodisciplina, como herramienta que ayuda en la consecución de labores trascendentales.

Si se considera la autodisciplina como un problema de mejoramiento continuo, se debe comprender que para el coach la auténtica meta no es conseguir que su grupo se comporte bien, sinoque cada miembro se responsable de su propia conducta. Los docentes suelen ser conscientes de los problemas educativos, por eso se plantea la auténtica naturaleza de la autodisciplina, la conducta competente para tener éxito en la escuela.

En este sentido, se menciona a Donoso, (2015), para quien las habilidades de autodisciplina ayudan a los docentes a planear y coordinar sus acciones, no perder de vista sus resultados. Estas habilidades les permiten controlar su propia conducta y generar la motivación y la buena disposición para actuar de la forma prescrita. Los alumnos utilizan las habilidades de autodisciplina para planear, gestionar y controlar con el fin de actuar de manera adecuada

Esta herramienta, de acuerdo con el citado autor se concentra en un propósito colosal, una motivación que sea tan grande, que solo el hecho de recordarla, alimente el deseo de conseguir lo que se quiere. Es una especie de obsesión positiva por lograr algo en pos del beneficio personal, de las personas, de una empresa o del mundo, sin detenerse ante los obstáculos que se puedan encontrar.

También, Guillermo y Alonzo (2010) consideran la autodisciplina como una herramienta transversal que facilita los procesos de motivación y persistencia y que por tanto permite lograr el éxito en cualquier emprendimiento, prácticamente en piloto automático. La autodisciplina es una de las grandes herramientas para el desarrollo personal. Por supuesto tampoco permite la solución a todos los problemas, sin embargo, coadyuva ala búsqueda de soluciones bastante significativas; proporcionando alternativas de solución de problemas, convirtiéndose en una herramienta poderosa al combinarla con otras como la pasión, el planeamiento y el fijarse metas específicas, ya que para Salazar y Molano (2013:34), la autodisciplina es la habilidad que tienes para tomar acción, sin importar el estado emocional, siendo muy beneficiosa para equipos de trabajo.

Sobre esto se infiere que su objetivo es fomentar una atmosfera de autodisciplina para aprender adesenvolverse y a imponerse una disciplina de forma más autónoma responsabilizándose más de su trabajo. Donde el primer requisito para la autodisciplina es hacer las cosas bien, es decir, que involucre resultados positivos de acuerdo a nuestro propósito, y que la misma es flexible y se puede adaptar de acuerdo al logro de resultados que se requiere.

\section{Reflexiones finales}

El coaching como herramienta gerencial, trae consigo elementos representativos tanto para la formación de líderes de alto desempeño, como para la concreción de entrenamientos que contribuyan al desarrollo personal, profesional e incluso social de quienes lo asumen como filosofía de vida, siguiendo sus preceptos de manera continua.

Por otro lado, se debe mencionar 
el coaching que se convierte en esencia fundamental para superar situaciones adversas en una sociedad que demanda grandes compromisos y exigencias. Convirtiéndose cada actuación, en un sentir responsable con la realidad circundante con la que interactúan las organizaciones,

Así, en el campo gerencial surge como herramienta que coadyuva a los niveles directivos a lograr desempeños exitosos en su personal y en su organización, centrándose en el desarrollo de competencias, conductas, habilidades y actitudes que permitan la potenciación de recursos, a la vez que propicien una mejor calidad de vida y mayor satisfacción con la práctica de la actividad gerencial cotidiana.

\section{Referencias Bibliográficas}

\section{Álvarez, G (2011). Cómo hacer una investigación cualitativa. EditrorialPaidos Educador. Buenos Aires-Argentina.}

Amador, P. (2009). El juego de la rueda de la vida. Editorial LID. México.

Aragón (2007). Coaching Educativo. Federación AECOP. Disponible: http://aecop.net/. Consultado 13/04/2016.

Arellano, E. José (2003). Epistemología de la Investigación Cuantitativa y Cualitativa. Editorial Trillas. México.

Arias, F. (2006). El Proyecto de Investigación. Introducción a la Metodología Científica. Editorial Episteme. Caracas, Venezuela.

BarberaAlbalat, V. (2010). La responsabilidad. Cómo educar en la responsabilidad. Aula XXI, Editorial Santillana. Madrid- España.

Barraza Macías, A.; Acosta Chávez, M. (2008). Compromiso organizacional de los docentes de una institución de educación media superior Innovación Educativa, Revista Arbitrada Volumen 8, Número 45, octubre-diciembre. Instituto Politécnico Nacional Distrito Federal, México.

Barbera Vicente. (2010). Organización de los Centros Educativos. Instituto para la Cooperación Educativa. España.

Bayo, S. (2014). Desarrollo de la confianza y la credibilidad en el coaching. Publicado en desarrollo coaches, negocio coaching, vende tu coaching, 15 mayo 2014. Disponible en:http://www.sorayabayo.com. Consultada: 04/12/15.

Bennett, M. (2013), Autoconocimiento. Ediciones Paidos. México.

Bernal, Cesar. (2008). Metodología de la investigación. Editorial Pearson. México.

Bisquerra, R. (2008). Coaching. Un reto para los orientadores. Revista de Orientación Psicopedagógica, Número 19. México.

Bou, J. F (2012). Coaching para docentes: El desarrollo de habilidades en el aula ( $3^{a}$ ed.) Editorial Alicante. Club Universitario. España.

Calvo Verdu, Miguel. (2008). Formación Profesional ocupacional. (FPO). Editorial Mad, S.L. España.

Certo, Samuel (2009). Administración moderna. Editorial McGraw-Hill. México.

Colorado Laguna, R. y Lillian Corcino, M. (2014). Percepción y receptividad al proceso de coaching como componente de un programa de desarrollo profesional para maestros de escuela primaria. Editorial Paradigma.Universidad de Puerto Rico, Río Piedras. 
Daft, Richard y Marcic, Dorothy (2006). Introducción a la Administración. Cuarta edición. International Thompson Editores. México.

Denzin, D. Norma (2008).El campo de la investigación cualitativa, Volumen 1. Editorial GEDISA. México.

Dessler, Gary. (2009). Administración del personal. Pearson Educación, 2001. México.

Diccionario de la Real Academia Española (2016). Diccionario Panhispánico de Dudas. Editorial Santillana. España.

Díaz B. Ángel. (2008). Coaching educativo: modelo para el desarrollo de competencias intra e interpersonales. Universidad de Valladolid. Colombia.

Donoso, José A. (2015).Competencias profesionales: Análisis conceptual y aplicación profesional. Conferencia Departamento de Métodos de Investigación y Diagnóstico en Educación. Universidad de Barcelona- España.

Eliott, Jhon. (2009). La investigación-acción en educación. Editorial Ediciones Morata. Madrid.

Escuela Básica Estatal Monseñor Álvarez (2013-2014). Manual de Convivencia Escolar. Mara-Zulia-Venezuela.

Fedor Eduardo (2010). Modelo para la gestión estratégica de la calidad total. Editorial EAE,. México.

Finol, M. y Camacho, H. (2010). El proceso de investigación científica. Colección libro de texto. $2^{\mathrm{a}}$ Edición. Editorial Ediluz. Maracaibo-Venezuela.

Gaete, R. (2011). La responsabilidad social universitaria como desafío para la gestión estratégica de la Educación Superior: el caso de España, Revista de Educación, №355. Dis- ponible: http://www.revista.educacion. Consultada: 14/04/2016.

García, B. (2011).Educación, integración o exclusión de la diversidad cultural? Ediciones Nina Kressova.

Goleman, Daniel (2000). La inteligencia emocional en la empresa. Vergara Editor. Tercera edición. Buenos Aires-Argentina

González Tovar, Manuel (2012). El coaching en la escuela. Editorial Trillas. México.

González, O; León,Joldry yGonzález, L. (2012), Modelo de Gerencia Académica, a través del Coaching docente en las Asesorías Académicas.

Griffin, R. (2011). Administración. Editorial Cengage Learning. México.

GuédezVictor (2008). Ser confiable. Responsabilidad Social u reputación empresarial. Editorial Planeta. Caracas-Venezuela.

Guillermo y Alonzo (2010). Medios de enseñanzas. Material para el autoaprendizaje. Ediciones: Universidad Autónoma de Yucatán.

Gutiérrez, E. (2010), Competencias gerenciales. Habilidades, conocimientos, aptitudes. Eco-ediciones. Bogotá, Colombia.

Hernández R.; Fernández, C. y Baptista, P. (2010) Metodología de la Investigación. Cuarta Edición. Editorial McGraw-Hill Interamericana de México, México.

Hurtado, J. (2008). El Proyecto de Investigación. Comprensión Holística de la Metodología y la Investigación. Ediciones Quirón. Caracas, Venezuela.

Kouzes, J. y Posner, B. (2006). Credibilidad. Cómo los líderes la obtienen y la pierden y proqué la gente la demanda. Ediciones GRANICA S.A. 
Disponible en: https://books.google. co.ve. Consultado: 23/5/2016.

Lagomarsino, R. (2003) Compromiso organizacional. Revista de Antiguos Alumnos, año VI, núm. 2, 2003, Universidad de Navarra. España.

Larez, Henríquez. (2008).Aprendizaje y métodos de docencia avanzada. Editorial Trillas. México.

López, O. (2009). Coaching educativo: Reflexiones sobre el papel del Coaching en la formación de la comunidad educativa. Revista el Rincón del coach. Disponible en: http://www.tisoc21sl.com. Consultado: $18 / 03 / 16$.

Lorenzo Delgado, M. (2014), La función de liderazgo de la dirección escolar: una competencia transversal. Enseñanza, Universidad de Granada. España.

Lussier, R. y Achua, C. (2005). Liderazgo. Teoría, Aplicación y Desarrollo de Habilidades. Editorial Thomson Learning. México.

Luthe García, Rodolfo (2006). El líder. Cómo formar a líderes del siglo XXI. Editorial Trillas. México.

Martínez, Miguelez (2009). La capacidad creadora y sus implicaciones para la metodología de la investigación. Departamento de Psicología Universidad Central de Venezuela. Caracas.

Máñez, C., Navarro, B. y Bou, J F. (S/A). Coaching para docentes el desarrollo de habilidades en el aula. Central Sindical Independiente y de Funcionarios (CSI-F).

Méndez, C. (2009). Metodología. Diseño y Desarrollo del Proceso de Investigación. Tercera Edición. Editorial McGraw-Hill Interamericana, S.A. Colombia.

Ministerio del poder Popular para la ed- ucación (2014). Líneas Estratégicas en el Marco del Proceso de transformación Educativa 20142015.

Molano, C. Mauricio. (2005)Coaching en acción: cómo desarrollar equipos de ventas de alta competitividad. Editorial McGraw-Hill. México.

Morales, L. (2012). Coaching como técnica para el desarrollo del talento humano en educación media general. Universidad Rafael Urdaneta. Maracaibo-Venezuela.

Morín, Edgar. (2008). Introducción al pensamiento complejo. Editorial Gedisa. Barcelona-España.

Murcia Florián, J. (2006): Investigar para cambiar. Editorial Magisterio. Bogotá-Colombia.

Obiols, M. y Giner, A. (2011). El modelo educativo de Bolonia y competencias docentes: Aportaciones desde el coaching educativo. Universidad de Valle Dolí. Colombia.

Odris González, Joldry León y Lislie González (2012). Modelo de Gerencia Académica, a través del Coaching docente en las Asesorías Académicas. Revista Encuentro Educacional. Volumen 19; Mayo Agosto. España.

Parra, R. (2012). La nueva escuela. Editorial Plaza y Jamés. Colombia.

Pascal, Debordes (2000). Coaching. Entrenamiento eficaz de los comerciales. Como motivar y hacer progresar a la fuerza de ventas. Editorial Gestión 2000. México.

Pelekais, C; Ferrer, J; Cruz, A. y Romero, D. (2007), Responsabilidad social, compromiso de las organizaciones. Venezuela. Ediciones Astro Data S.A. 
Pérez, A. (2008).Investigación Cualitativa. Retos e Interrogantes. Editorial La Muralla. Madrid-España.

Pereira, Eduardo (2009). Planificación organizacional. Editorial GRAO. Venezuela.

Piñeiro, M; Martínez, M. y Guillén, E. (2013).Planificación y organización de la docencia. Enseñar y aprender mediante coaching educativo. Editorial Univest 2013. Girona.

Red Española de Información sobre Educación -Eurydice- (2015), Informe Comisión Europea. España.

Richardson, P. (2005). Coaching Personal. Guía práctica para descubrir y desarrollar sus propias capacidades. Ediciones Parramon S.A. Barcelona España.

Risquez, G; Pereira, B. y Fuenmayor, E. (2009). Metodologpia de la investigación. Manual Teórico Práctico. Editorial $\mathrm{N}$ y N Publicaciones. Maracaibo-Venezuela.

Robbins, S. y Coulter, M. (2010). Administración. Décima Edición. Editorial Prentice-Hall. México.

Rodríguez M. Ernesto A. (2011) Metodología de la Investigación. Edición Universidad Juárez Autónoma de Tabasco. México

Rojas, Belkys. (2010), Investigación Cualitativa. Fundamentos y praxis. Editorial FEDUPEL. Caracas-Venezuela.

Ruiz, José Ignacio. (2012). Como elaborar un Proyecto de Investigación Social. Editorial Narcea, S.A. Madrid España.

RyderGuy. (2015), Agenda de Desarrollo hacia 2030: Un gran avance para el mundo del trabajo. Organización Internacional del Trabajo (OIT), Ginebra.
Sabino, Carlos. (2007). El proceso de investigación. Una introducción teórico-práctica. Editorial Panapo.

Salas, E. y Mendoza, A. (2005).La Representación social de la calificación educativa. Editorial EAE. México.

Salazar T. Gilberto y Molano Camacho, M. (2000). Coaching en acción: como desarrollar equipos de ventas de alta competición. Editorial Mc Graw-Hill. Colombia.

Sánchez Mirón, B. y BoronatMundina, J. (2014). Coaching Educativo: Modelo para el desarrollo de competencias intra e interpersonales. Educación XXI. España.

Seijas, F. (2003) Investigación por muestreo. Ediciones de FACES. Universidad Central de Venezuela. Caracas-Venezuela.

Saphier, J. y West L. (2010).Cómo los entrenadores pueden maximizar el aprendizaje del estudiante. Editorial Phi Delta. España.

Tamayo y Tamayo, Mario (2010). El proceso de la investigación científica. Editorial Limusa Noriega. México.

Urdaneta, S. (2012).Responsabilidad social y ética gerencial en las instituciones educativas del Municipio Maracaibo. Universidad Rafael Urdaneta. Maracaibo-Venezuela.

Vallejo, R.; Finol de Franco, M.; Camacho, H. (2010). Responsabilidad social como principio para la gestión del proyecto educativo integral comunitario. Multiciencias, Volumen 10, Número. 1, enero-abril, Universidad del Zulia. Punto Fijo-Venezuela.

Varona, Madrid, Federico. (2011). Conceptualización y supervisión de la comunicación y el compromiso organizacional. Editorial DIA-LOGOS. España. 
pp. $904-921$

Revista Venezolana de Gerencia, Año 24 N 87, 2019

Vinardi, Libio (2016). Autoconocimiento -

Nuevos Enfoques (Biopsicoenergéti-

ca, Healing, Biorritmología y Sistema

Isotérico). Editorial Youcanprint. Es-

paña.

- $\quad$ Esta obra está bajo una licencia de Creative Commons Reconocimiento-NoComercial- Compartirlgual 3.0 Unported. http://creativecommons.org/licenses/by-nc-sa/3.0/deed.es_ES 